\title{
Nightmares and Posttraumatic Stress Disorder (PTSD)
}

\author{
Rebecca L. Campbell ${ }^{2}$ Anne Germain ${ }^{1}$
}

Published online: 22 March 2016

(C) Springer International Publishing AG 2016

\begin{abstract}
Nightmares are a unique feature of posttraumatic stress disorder (PTSD). Although nightmares are a symptom of PTSD, they have been shown to independently contribute to psychiatric distress and poor outcomes, including heightened suicidality and suicide. Nightmares are often resistant to recommended pharmacological or psychological PTSD treatments. Fortunately, nightmare-specific treatments are available, and improvements in nightmares are associated with clinically significant improvements in sleep quality and severity of daytime PTSD symptoms. The recent literature on the characteristics, neurophysiology, and treatment of nightmares in the context of PTSD is reviewed. Recent findings on the neurophysiological correlates of nightmares and more generally, dreaming, are also discussed here as they suggest novel directions for understanding the mechanisms underlying nightmares comorbid with PTSD and potential novel treatment approaches.
\end{abstract}

Keywords Nightmares $\cdot$ PTSD $\cdot$ Sleep $\cdot$ Dreaming $\cdot$ Trauma $\cdot$ Stress

This article is part of the Topical Collection on Parasomnias

Anne Germain

germax@upmc.edu

Rebecca L. Campbell

campbellr13@upmc.edu

1 University of Pittsburgh Medical Center, Western Psychiatric Institute and Clinic, 3811 O'Hara Street, Sterling Plaza 235, Pittsburgh, PA 15213, USA

2 Department of Psychiatry, University of Pittsburgh School of Medicine, 3811 O'Hara Street, Sterling Plaza 240, Pittsburgh, PA 15213, USA

\section{Introduction}

Nightmares are a hallmark of posttraumatic stress disorder (PTSD). In the context of PTSD, nightmares are typically conceptualized as dysphoric dreams that completely or partially depict aspects of traumatic events. Nightmares are associated poor health and treatment outcomes such as poor sleep quality, daytime distress, and higher risk of suicide. Treatments targeting nightmares can also improve sleep and daytime symptoms of anxiety and depression.

In this article, we review the diagnostic criteria for nightmare disorder, recent studies on the content and characteristics and their relationship to functional outcomes, as well as new animal research that can inform our understanding of the pathophysiology underlying nightmares. New findings regarding established and novel psychological and pharmacological approaches for the treatment for nightmares with comorbid PTSD are also summarized here.

\section{Diagnostic Criteria for Nightmare Disorder}

The third edition of the International classification of sleep disorders (ICSD-3) [1, 2] has recently redefined nightmares as "repeated occurrences of extended, extremely dysphoric, and well-remembered dreams that usually involve threats to survival, security, or physical integrity" [1]. Contrary to several other parasomnias, the sleeper rapidly regains alertness and orientation upon awakening from a nightmare. Nightmare disorder is characterized by dysphoric dreams that perturb sleep consolidation and daytime functioning. While nightmares are developmentally common in young children, traumatic events and pharmacological agents are the most commonly identified precipitating factors in adults. 
Nightmares are one of the re-experiencing symptoms of PTSD [3], and affect as many as $50 \%$ of trauma-exposed adults. There is early evidence to suggest that men are more likely to experience nightmares as a symptom of PTSD than women. Males deployed in support of Operation Enduring Freedom (October, 2010) or Operation Iraqi Freedom (March, 2003) are more likely to experience nightmares, numbing, and hyper-vigilance than their female counterparts who experience more concentration problems and distress with reminders [4•]. Whether or not the same pattern holds in the civilian population or with nightmares without comorbid PTSD has yet be determined.

\section{Content and Characteristics of Nightmares Comorbid with PTSD}

Posttraumatic nightmares typically involve themes and sensory input related to a specific traumatic event. Harb and colleagues [5••] studied a sample of 48 combat-exposed Vietnam veterans to find a relationship between dream content and treatment outcomes of image rehearsal therapy (IRT), an intervention that promotes mastery over recurrent nightmares by rehearsing modified versions of the disturbing nightmare [6, 7]. Reports of olfactory experiences during original nightmares predicted a smaller reduction in sleep disturbances, possibly due to the link between odor perception and emotional memory. Harb and colleagues reasoned that experiencing smells may indicate intensity of a nightmare because of the rudimentary nature of the brain systems responsible for olfactory processing [5]. If that is the case, nightmare characteristics such as sensory experiences may inform patient prognosis.

Traumatic themes may also be subjected to cultural differences. Hinton and colleagues [8•] interviewed Cambodian refugees that survived and lost loved ones in the Pol Pot genocide (1975-1979) about the frequency, content, and meaning of dreams. While investigators did not refer to nightmares specifically, dreams described as "deeply upsetting" by all participants but one of the participants "awoke sobbing" paired with sadness and fear [8•]. In this cultural context, dreams themselves could be traumatic for the dreamer. When a deceased loved one appears in dreams, it means he or she is suffering and begging the dreamer for help [8•]. Such dreams could also be spiritual attack on the dreamer themselves that are thought to result in severe illness or death [8•]. The number of dreams in the past month correlated with PTSD symptom severity as measured by the PTSD checklist $(r=-0.53, p<0.01)$. More exploration into cultural differences in nightmare content is warranted because treatment approaches could be modified to meet the needs of patients.

Nightmares are not only associated with higher severity of PTSD symptoms at baseline as demonstrated in a study of 80 combat veterans by Pigeon et al. [9•]; nightmares may also predict higher PTSD severity at a six-month follow-up. In a study by Van Liempt et al. [10•], 453 Dutch soldiers were interviewed before and six months after a deployment to Afghanistan. Nightmares prior to deployment predicted PTSD at post deployment. Thus, targeting the treatment nightmares prior to or early after deployment offers a strategy to enhance resilience and/or mitigate the risk of poor deployment-related psychiatric symptoms and disorders.

\section{Understanding the Pathophysiology of Nightmares Through Basic Research}

Studying nightmares in the sleep laboratory or using sleep neuroimaging methods is highly challenging as these episodes cannot be reliably experimentally induced and rarely occur under laboratory conditions, even in individuals with chronic and frequent nightmares. Thus, animal models focused on the impact of trauma-like events on sleep and behaviors can provide some novel insights into the potential pathways underlying the pathophysiology of nightmares comorbid with PTSD.

One such study was conducted by Vanderheyden and colleagues [11••], and collected electroencephalogram (EEG) data before and after exposing rats to single prolonged stress, and measured freezing behaviors during a fear-learning task to determine PTSD-like symptom severity. They observed that an increase in REM sleep from baseline, and a reduction of sigma and theta waves in transition to REM was associated with increased freezing behavior during fear recall. Because nightmares are associated with an increase in REM sleep within eight hours of stress exposure, nightmares may interfere with fear extinction $[11 \bullet \cdot$. When applied in a real world context, nightmares could exacerbate the development of PTSD symptoms or hinder PTSD treatment.

Bin Yu et al. [12••] sought to understand the differences in neural circuitry between rats that startled awake by assumed "nightmares" related to either physical trauma by a foot shocks, or psychological trauma by witnessing physical trauma. Twenty-one days after the initial trauma, the rats were put back in the communication box, without the foot shocks, after which, six hours of EEG was recorded. EEG analyzed one minute before the startled awakenings revealed higher delta power density and lower theta, alpha, and beta power density on the physically stressed rats while psychologically stressed rats had no changes in EEG analysis. Upon startled awakenings, animals were terminated and necropsies were performed. Neural circuits also differed between groups. Psychologically stressed rats experienced decreased activity in the granular and dysgranular insular cortex and increased activation in the temporal association cortex. Physically stressed rats displayed increases in the secondary somatosensory and primary auditory cortices. Both groups exhibited inactivation of 
the infralimbic prefrontal cortex and ventral anterior cingulate cortex with activation in the amygdala. These findings suggest that at least in animals, not only can the underlying mechanisms of PTSD and nightmares during sleep could be identified but also that they may differ depending on the type of trauma experienced [12••]. If types of trauma impact the brain differently, future studies should investigate the extent to which different types of trauma may be associated with distinct physiological correlates and treatment outcomes.

\section{Treatment of Nightmares}

Much improvement is still needed in rising awareness about the clinical relevance and the availability of effective nightmare treatments. Two recent surveys of over 1500 adults [13*0] found that $7 \%$ of respondents endorsed clinically significant nightmares, as defined by a score $>10$ on the disturbing dreams and nightmare severity index (DDNSI) [14], a questionnaire that measures frequency and intensity of nightmares, with scores of 10 or higher indicating a nightmare disorder [14]. Among adults who endorsed clinically significant nightmares, only 11 and $38 \%$ reported that they had discussed nightmares with a healthcare provider. More importantly, only one-third of adults who endorsed clinically significant nightmares believed that nightmares were a treatable condition. The authors proposed that screening for nightmares in a more consistent manner among providers as well as education may improve utilization of evidence-based nightmare treatments, as well as mitigate the widespread belief that nightmares are not a treatable condition [13・•].

Because nightmares are a symptom of PTSD and are often resistant to first-line PTSD treatments, much of the research in the last decade has targeted the treatment of nightmares comorbid with PTSD or trauma-related disorders. The impact of recommended psychological treatments of PTSD on nightmares and overall sleep quality has been more closely studies in recent years. The effectiveness of nightmare-focused treatments such as imagery rehearsal therapy and prazosin has also been re-evaluated in recent studies. Recent findings with psychological approaches and pharmacological agents are summarized below.

\section{Psychological Treatments}

Gutner and colleagues [15•] reported significant improvements in subjective sleep measures over the course of cognitive processing therapy, a treatment method that focuses on rectifying maladaptive emotional processing [16] and prolonged exposure, that uses imagined and in vivo exposure to facilitate fear extinction [17] in an intent to treat sample of 171 female victims of rape. Improvements were maintained at follow-up five to 10 years after treatment. While there was a clinically significant improvement in PTSD symptoms reported in an earlier publication [18], sleep disturbances did not reach full remission. Although daytime PTSD symptoms improved without full remission of sleep disturbances, healthy sleep improves quality of life, and treating nightmares and disturbed sleep should not be neglected.

Sloan et al. [19•0] released a meta-analysis that reported the large effects $(d=0.71,95 \%$ CI $[0.51,0.91])$ within group PTSD treatment compared to waitlist comparison $(d=0.56$, $95 \%$ CI $[0.31,0.82])$ on PTSD symptoms. The 16 included publications were all between-condition randomized designs examining group treatment of PTSD in an outpatient sample over the age of 18 . These effect sizes were similar to those reported by Casement and Swanson [20••]. Specifically, Casement and Swanson conducted a meta-analysis that included 13 studies published before July $30^{\text {th }}$, 2011 that focused on IRT or IRT and cognitive behavioral therapy for insomnia (CBTi). A majority of the studies used a group format with the number of sessions ranging from three to 10 . IRT had a large effect on frequency of nightmares $(\mathrm{ES}=0.6995 \%$ CI $[0.50,0.88])$ sleep quality ( $\mathrm{ES}=0.6895 \% \mathrm{CI}[0.34,1.03])$, and PTSD symptoms (ES $=0.7295 \% \mathrm{CI}[0.54,0.89])$. IRT combined with CBT had significant improvement of sleep quality $(p<0.001)$ but not PTSD symptoms $(p=0.87)$ or nightmares $(p=0.13)[21 \bullet \bullet]$.

Schagen et al. [22•] studied nightmare reduction in a population with a range of psychiatric diagnoses. Participants were assigned to an IRT group or treatment as usual group. They found significant improvements across PTSD, anxiety, and depression in the IRT group at post-treatment, but only PTSD scores had significant improvements at a three-month follow-up whereas depression and anxiety did not. IRT shows potential in comorbidities such as anxiety and depression; however, the impact of treating nightmares has on PTSD symptomology speaks to the entwined nature of PTSD daytime symptoms and the traumatic nightmares that often accompany them.

While treating nightmares across psychiatric disorders seems promising, nightmares associated with PTSD present a unique set of challenges. Thunker et al. [23•] looked at the effectiveness of IRT in adults with nightmares and PTSD, depression, or no comorbidities. While IRT was effective across all three groups, the effect was less pronounced in the PTSD group. They speculate that this could be due to a higher number of nightmares at baseline or more psychological strain associated with PTSD and PTSD treatment.

In the Thunker et al. study [23•], the participants with PTSD were divided into IRT and TAU groups. The IRT group had a reduction in nightmare frequency but not in anxiety or number of awakenings. Thunker et al. hypothesize that the stressful nature of PTSD therapy may impact the effectiveness of IRT. Gehrman et al. [24••] observed that daytime stressors, theoretically such as stresses of treatment for PTSD, were associated with more nightmares, nightmare-related distress, 
and longer sleep onset latency on the nights that nightmares occurred. If treatment of PTSD interferes with the treatment of nightmares and vice versa as demonstrated in the above studies, the order of treatment administration should be considered.

In contrast, a study by Margolies et al. [25•] compared a group of post $9 / 11$ veterans that received CBT with IRT to a waitlist group. While the treatment group improved in subjective and objective sleep measures, PTSD, depression, and distress, there was no change in nightmares. Margolies et al. speculated that this could be attributed to differences in a military population or possible non-compliance. Treatment adherence should be studied if IRT is to truly be considered effective.

\section{Pharmacological Treatments of Nightmares}

\section{Alpha-1 Noradrenergic Antagonists}

Although the etiology and pathophysiology of nightmares remain unknown, heightened noradrenergic tone during sleep and especially rapid-eye movement (REM) sleep has been proposed as a potential mechanisms underlying nightmares in the context of PTSD [26, 27].

Prazosin is an alpha-1 noradrenergic antagonist that crosses the blood brain barrier, and that may reduce nightmares and sleep disturbances that characterize PTSD [27-31]. Based on the extensive review of open trials and placebo-controlled studies conducted in military veterans and civilians with nightmares PTSD, the American Academy of Sleep Medicine (AASM) recommended prazosin as an evidencebased treatment of nightmares, especially in the context of PTSD [32]; see also: [33-35] for recent reviews.

Since the AASM recommendations were released in 2010, a number of randomized controlled trials have been conducted [36-38], which have generally supported the effectiveness of prazosin for nightmares comorbid with PTSD. In a prazosin augmentation study conducted in active duty service members with combat-related nightmares and PTSD, Raskind and colleagues [37] showed clinically meaningful improvements in nightmares, sleep quality, PTSD severity, and overall functioning relative to placebo. In this trial, a mid-morning dose of up to $5 \mathrm{mg}$ for men and $2 \mathrm{mg}$ for women was augmented the bedtime dose (up to $20 \mathrm{mg}$ for men and $10 \mathrm{mg}$ for women), and improvements in daytime symptoms of hyperarousal, but not of re-experiencing, were also detected. Thus, prazosin may not only impact arousal during sleep but may also have benefits for daytime symptoms of PTSD or other arousalrelated behaviors [39-41].

Ahmadpanah and colleagues [36] conducted an eight-week randomized clinical trial to compare prazosin and hydroxyzine to a placebo condition on measures of nightmares, sleep quality, and PTSD symptom severity in sample of 100 adult civilians. Hydroxyzine is a histaminergic receptor blocker that has anxiolytic and sedative properties and may be effective in the treatment of anxiety disorders [42]. Both active treatments were associated with improvements in nightmares, overall sleep quality, and PTSD symptomatology, and the magnitude of improvements was slightly greater for the prazosin group.

Nirmalani-Gandhy and colleagues have described the cases of four combat veterans (three men and one woman) with chronic PTSD who failed a prazosin trial, but who showed improvements in nightmares with terazosin, another alpha-1 noradrenergic antagonist [43]. Effective terazosin doses ranged between $2 \mathrm{mg}$ titrated up to $10 \mathrm{mg}$. In one patient, augmentation of nightly terazosin doses with morning dose of $2 \mathrm{mg}$ improved symptoms of arousal. Of note, all four patients presented with comorbid psychiatric disorders and complex pharmacological profiles. This case series suggest that terazosin may offer a novel treatment approach for nightmares in the context of chronic PTSD, but controlled rigorous clinical trials in larger samples are required to establish the efficacy of terazosin.

\section{Cannabis and Synthetic Endocannabinoids}

The sedative, anxiolytic, and hypnotic effects of cannabis ( $\Delta^{9}$-tetrahydrocannabinol or THC) have contributed to the growing medical or recreational use of cannabis to cope with PTSD symptoms, including nightmares, among traumaexposed individuals [44]. Recent studies have found a positive association between cannabis use and severity of hyperarousal symptoms, including nightmares and sleep disturbances in veterans and civilian samples, e.g., [45-47]. In a cross sectional study of 170 adult cannabis users, Bonn-Miller and colleagues [48] found that sleep disturbance was an independent predictor of recent cannabis use in adults with PTSD. Others have found that poor sleep quality predicted impeded reduction in cannabis use following a self-guided attempt to quit in a sample of veterans [49] or contribute to relapse [50]. Studies on impact effects of cannabis using objective sleep measure methods are scarce, and generally find that cannabis disrupts sleep continuity [47]. Similar, the efficacy of cannabis in the treatment of nightmares comorbid with PTSD remains scarcely investigated (see for review [51]). Of note, different strains of cannabis may differentially impact nightmares and insomnia comorbid with PTSD [44].

There is some preliminary evidence that the synthetic endocannabinoid receptor agonist, nabilone, a cannabinoid 1 $\left(\mathrm{CB}_{1}\right)$ that is FDA-approved for chemotherapy-related side effects, may be effective to reduce nightmares in PTSD. Although nabilone has a profile similar to tetrahydrocannabinol (THC), it does not have the euphoric effects of cannabis. The use nabilone in PTSD is not new: Fraser [52] reported significant reduction or cessation of PTSD-related nightmares in Canadian soldiers with nightly doses of nabilone ranging 
between 0.2 and $4 \mathrm{mg}$. Like prazosin, discontinuation of nabilone can be associated with a recurrence of nightmares. Reductions in nightmares were associated with improvements in sleep quality and reduction in daytime re-experiencing symptoms. Reported side effects are generally minor and can include dizziness, lightheadedness, forgetfulness, and headache.

In a retrospective chart review, Cameron and colleagues recently [53] reported significant reduction in the number of nights with nightmares as well as overall improvements in sleep quality, daytime PTSD symptom severity, and overall functional in a sample of 90 inmates. Improvements in nightmares and sleep quality were rapidly detected ( $\leq 2$ weeks) and maintained over time. The potential benefits of nabilone for the treatment nightmares comorbid with PTSD was recently further highlighted in a double-blind, placebo-controlled cross-over study conducted by Jetly and colleagues [54] in a small sample of ten service members with PTSD. Compared to placebo, nabilone was associated with significant reductions in nightmare severity and improved overall PTSD symptom severity. In this trial, benefits were observed as doses between 0.5 and $3 \mathrm{mg}$, and the medication was well tolerated. Published reports to date suggest that nabilone is well tolerated at doses that range between 0.5 and $4 \mathrm{mg}$, and that common side effects include dizziness, headaches, dry month, and lightheadedness. One case of psychosis has been reported [53].

\section{Conclusion}

Nightmares are an underestimated sleep disorder and are a core feature of PTSD that requires targeted assessment and treatment. The recent changes and broadening of the diagnostic for nightmare disorder raise the possibility that nightmares comorbid with PTSD may also include a wider range of dysphoric content than the strict focus on trauma-related dreams currently stated for the diagnosis of PTSD. The content of nightmares comorbid with PTSD has yet to be fully investigated in large samples using prospective dream sampling methods. While a detailed content analysis may not be necessary for diagnosis or treatment of nightmares in PTSD, such analysis may provide novel directions for treatment options and underlying cognitive mechanisms.

Nevertheless, case reports and clinical trials focused on the treatment of nightmares have been consistent in recent years. These reports have mainly focused on the recommended and novel treatment approaches, within more heterogeneous samples including individuals with psychiatric comorbidities or varying forms of trauma. Nevertheless, treatment studies have generally been limited by the lack of data regarding participant adherence to treatment recommendations. Although the progress in psychological treatment options has been encouraging, no novel methods have been introduced. Novel pharmacological agents, including terazosin and synthetic endocannabinoid agents, may offer new treatments to target nightmares comorbid with PTSD. Larger randomized controlled trials are required to fully determine safety and efficacy.

\section{Compliance with Ethical Standards}

Conflict of Interest The authors declare that they have no conflict of interest

Human and Animal Rights and Informed Consent This article does not contain any studies with human or animal subjects performed by any of the authors.

\section{References}

Papers of particular interest, published recently, have been highlighted as:

- Of importance

•• Of major importance

1. American Academy of Sleep Medicine. International classification of sleep disorders. 3rd ed. Darien, IL: American Academy of Sleep Medicine; 2014.

2. American Academy of Sleep Medicine (AASM). American Academy of Sleep Medicine (AASM). www.aasmnet.org 2009

3. Narrow WE, Clarke DE, Kuramoto SJ, Kraemer HC, Kupfer DJ, Greiner L, et al. DSM-5 field trials in the United States and Canada, Part III: development and reliability testing of a cross-cutting symptom assessment for DSM-5. Am J Psychiatry. 2013;170:71-82.

4. King MW, Street AE, Gradus JL, Vogt DS, Resick PA. Gender differences in posttraumatic stress symptoms among OEF/OIF veterans: an item response theory analysis. J Trauma Stress. 2013;26: 175-83. doi:10.1002/jts.21802. Male OEF/OIF veterans with PTSD are more likely to experience nightmares than female veterans with PTSD.

5.• Harb GC, Thompson R, Ross RJ, Cook JM. Combat-related PTSD nightmares and imagery rehearsal: nightmare characteristics and relation to treatment outcome. J Trauma Stress. 2012;25:511-8. doi: $10.1002 /$ jts. 21748 . Sensory experiences during nightmares may predict severity of nightmares and treatment outcomes.

6. Krakow B, Hollifield M, Johnston L, Koss M, Schrader R, Warner $\mathrm{TD}$, et al. Imagery rehearsal therapy for chronic nightmares in sexual assault survivors with posttraumatic stress disorder: a randomized controlled trial. JAMA. 2001;286:537-45.

7. Forbes D, Phelps A, McHugh T. Treatment of combat-related nightmares using imagery rehearsal: a pilot study. J Trauma Stress. 2001;14:433-42.

8. Hinton DE, Field NP, Nickerson A, Bryant RA, Simon N. Dreams of the dead among Cambodian refugees: frequency, phenomenology, and relationship to complicated grief and posttraumatic stress disorder. Death Stud. 2013;37:750-67. doi:10.1080/07481187. 2012.692457. Dreams and nightmares are subjected to cultural interpretation and may be traumatic events in and of themselves, possibly contributing to PTSD severity. 
9. Pigeon WR, Campbell CE, Possemato K, Ouimette P. Longitudinal relationships of insomnia, nightmares, and PTSD severity in recent combat veterans. J Psychosom Res. 2013;75:546-50. doi:10.1016/ j.jpsychores.2013.09.004. Nightmares are related to higher PTSD severity at baseline.

10. van LS, van ZM, Westenberg H, Super A, Vermetten E. Impact of impaired sleep on the development of PTSD symptoms in combat veterans: a prospective longitudinal cohort study. Depress Anxiety. 2013;30:469-74. doi:10.1002/da.22054. Nightmares before deployment predicted the development of PTSD after deployment.

11.• Vanderheyden WM, George SA, Urpa L, Kehoe M, Liberzon I, Poe GR. Sleep alterations following exposure to stress predict fearassociated memory impairments in a rodent model of PTSD. Exp Brain Res. 2015;233:2335-46. doi:10.1007/s00221-015-4302-0. Nightmares interfere with fear extinction in stressed rats.

12.• Yu B, Cui SY, Zhang XQ, Cui XY, Li SJ, Sheng ZF, et al. Different neural circuitry is involved in physiological and psychological stress-induced PTSD-like "nightmares" in rats. Sci Rep. 2015;5: 15976. doi:10.1038/srep15976. Nightmares induced by varying forms of trauma in rats are associated with changes in neural circuitry. Changes in the brain differed between types of trauma.

13.• Nadorff MR, Nadorff DK, Germain A. Nightmares: under-reported, undetected, and therefore untreated. J Clin Sleep Med. 2015;11: 747-50. doi:10.5664/jcsm.4850. A small minority of nightmare sufferers actually seek treatment. Only one in three believe that nightmares are treatable.

14. Krakow BJ, Melendrez DC, Johnston LG, Clark JO, Santana EM, Warner TD, et al. Sleep dynamic therapy for Cerro Grande Fire evacuees with posttraumatic stress symptoms: a preliminary report. J Clin Psychiatry. 2002;63:673-84. doi:10.4088/JCP.v63n0804.

15. Gutner CA, Casement MD, Stavitsky GK, Resick PA. Change in sleep symptoms across cognitive processing therapy and prolonged exposure: a longitudinal perspective. Behav Res Ther. 2013;51: 817-22. doi:10.1016/j.brat.2013.09.008. Female rape victims maintained improvements in PTSD symptomology at a fiveto ten-year follow-up to cognitive processing therapy and prolonged exposure. Sleep disturbances did not fully remit.

16. Resick PA, Schnicke MK. Cognitive processing therapy for rape victims: a treatment manual (interpersonal violence: the practice series). First ed. Newbury Park: SAGE Publication, Inc., 1993.

17. Foa E, Hembree E, Rothbaum BO. Prolonged exposure therapy for PTSD: emotional processing of traumatic experiences therapist guide. New York: Oxford University Press, 2007.

18. Resick PA, Nishith P, Weaver TL, Astin MC, Feuer CA. A comparison of cognitive-processing therapy with prolonged exposure and a waiting condition for the treatment of chronic posttraumatic stress disorder in female rape victims. J Consult Clin Psychol. 2002;70:867-79. doi:10.1037/0022-006X.70.4.867.

19.• Sloan DM, Feinstein B, Gallagher MW, Beck JG, Keane TM. Efficacy of group treatment for posttraumatic stress disorder: a meta-analysis. Psychological Trauma Theory Research Practice and Policy. 2013;5:176-83. Group PTSD treatment demonstrated high effect sizes on PTSD symptoms.

20.• Casement $\mathrm{M}$, Germain A. Is group imagery rehearsal for posttrauma nightmares as good at reducing PTSD symptoms as group treatment for PTSD? Psychological Trauma Theory Research Practice and Policy. 2014;6:259. doi.org/10.1037. Group PTSD treatment effect sizes [19] were similar to IRT or IRT and CBT effect sizes [21].

21.• Casement MD, Swanson LM. A meta-analysis of imagery rehearsal for post-trauma nightmares: effects on nightmare frequency, sleep quality, and posttraumatic stress. Clin Psychol Rev. 2012;32:56674. doi:10.1016/j.cpr.2012.06.002. A meta-analysis of IRT or IRT and CBT demonstrated that IRT had a large effect on PTSD symptoms and nightmares while IRT and CBT had large effects on sleep disturbances but not PTSD.

22. van Schagen AM, Lancee J, de Groot IW, Spoormaker VI, van den Bout J. Imagery rehearsal therapy in addition to treatment as usual for patients with diverse psychiatric diagnoses suffering from nightmares: a randomized controlled trial. J Clin Psychiatry. 2015;76: e1105-13. doi:10.4088/JCP.14m09216. Patients with nightmares and comorbidities were given IRT. The IRT group had significant improvements across PTSD, anxiety and depression at post-treatment, but only PTSD patients had significant improvement at follow up.

23. Thunker J, Pietrowsky R. Effectiveness of a manualized imagery rehearsal therapy for patients suffering from nightmare disorders with and without a comorbidity of depression or PTSD. Behav Res Ther. 2012;50:558-64. doi:10.1016/j.brat.2012.05.006. IRT effective across Nightmares with PTSD, depression and no comorbidities however it was less pronounced in PTSD group.

24.• Gehrman PR, Harb GC, Cook JM, Barilla H, Ross RJ. Sleep diaries of Vietnam War veterans with chronic PTSD: the relationships among insomnia symptoms, psychosocial stress, and nightmares. Behav Sleep Med. 2015;13:255-64. doi:10.1080/15402002.2014. 880344. Daytime stressors were associated with more nightmare distress and sleep onset latency on the nights with nightmares.

25. Margolies SO, Rybarczyk B, Vrana SR, Leszczyszyn DJ, Lynch J. Efficacy of a cognitive-behavioral treatment for insomnia and nightmares in Afghanistan and Iraq veterans with PTSD. J Clin Psychol. 2013;69:1026-42. doi:10.1002/jclp.21970. CBT and IRT improved PTSD symptoms and sleep quality but not nightmares possibly because of non-compliance or a military population.

26. Arnsten AF, Raskind MA, Taylor FB, Connor DF. The effects of stress exposure on prefrontal cortex: translating basic research into successful treatments for post-traumatic stress disorder. Neurobiol Stress. 2015;1:89-99. doi:10.1016/j.ynstr.2014.10.002.

27. Raskind MA, Dobie DJ, Kanter ED, Petrie EC, Thompson CE, Peskind ER. The alpha1-adrenergic antagonist prazosin ameliorates combat trauma nightmares in veterans with posttraumatic stress disorder: a report of 4 cases. J Clin Psychiatry. 2000;61:129-33.

28. Peskind ER, Bonner LT, Hoff DJ, Raskind MA. Prazosin reduces trauma-related nightmares in older men with chronic posttraumatic stress disorder. J Geriatr Psychiatry Neurol. 2003;16:165-71. doi: 10.1177/0891988703256050.

29. Raskind MA, Peskind ER, Kanter ED, Petrie EC, Radant A, Thompson CE, et al. Reduction of nightmares and other PTSD symptoms in combat veterans by prazosin: a placebo-controlled study. Am J Psychiatry. 2003;160:371-3. doi:10.1176/appi.ajp. 160.2.371

30. Raskind MA, Thompson C, Petrie EC, Dobie DJ, Rein RJ, Hoff DJ, et al. Prazosin reduces nightmares in combat veterans with posttraumatic stress disorder. J Clin Psychiatry. 2002;63:565-8.

31. Taylor F, Raskind MA. The alpha1-adrenergic antagonist prazosin improves sleep and nightmares in civilian trauma posttraumatic stress disorder. J Clin Psychopharmacol. 2002;22:82-5.

32. Aurora RN, Zak RS, Auerbach SH, Casey KR, Chowdhuri S, Karippot A, et al. Best practice guide for the treatment of nightmare disorder in adults. J Clin Sleep Med. 2010;6:389-401.

33. Writer BW, Meyer EG, Schillerstrom JE. Prazosin for military combat-related PTSD nightmares: a critical review. J Neuropsychiatry Clin Neurosci. 2014;26:24-33.

34. Kung S, Espinel Z, Lapid MI. Treatment of nightmares with prazosin: a systematic review. Mayo Clin Proc. 2012;87:890-900. doi:10.1016/j.mayocp.2012.05.015.

35. Khachatryan D, Groll D, Booij L, Sepehry AA, Schutz CG. Prazosin for treating sleep disturbances in adults with posttraumatic stress disorder: a systematic review and meta-analysis of 
randomized controlled trials. Gen Hosp Psychiatry. 2015. doi:10. 1016/j.genhosppsych.2015.10.007

36. Ahmadpanah M, Sabzeiee P, Hosseini SM, Torabian S, Haghighi $\mathrm{M}$, Jahangard L, et al. Comparing the effect of prazosin and hydroxyzine on sleep quality in patients suffering from posttraumatic stress disorder. Neuropsychobiology. 2014;69:235-42. doi:10. 1159/000362243.

37. Raskind MA, Peterson K, Williams T, Hoff DJ, Hart K, Holmes H, et al. A trial of prazosin for combat trauma PTSD with nightmares in active-duty soldiers returned from Iraq and Afghanistan. Am J Psychiatry. 2013;170:1003-10. doi:10.1176/appi.ajp.2013. 12081133

38. Germain A, Richardson R, Moul DE, Mammen O, Haas G, Forman $\mathrm{SD}$, et al. Placebo-controlled comparison of prazosin and cognitivebehavioral treatments for sleep disturbances in US Military Veterans. J Psychosom Res. 2012;72:89-96. doi:10.1016/j. jpsychores.2011.11.010.

39. Olson VG, Rockett HR, Reh RK, Redila VA, Tran PM, Venkov $\mathrm{HA}$, et al. The role of norepinephrine in differential response to stress in an animal model of posttraumatic stress disorder. Biol Psychiatry. 2011;70:441-8. doi:10.1016/j.biopsych.2010.11.029.

40. Rasmussen DD, Alexander LL, Raskind MA, Froehlich JC. The alpha1-adrenergic receptor antagonist, prazosin, reduces alcohol drinking in alcohol-preferring (P) rats. Alcohol Clin Exp Res. 2009;33:264-72. doi:10.1111/j.1530-0277.2008.00829.x.

41. Simpson TL, Saxon AJ, Meredith CW, Malte CA, McBride B, Ferguson LC, et al. A pilot trial of the alpha-1 adrenergic antagonist, prazosin, for alcohol dependence. Alcohol Clin Exp Res. 2009;33:255-63. doi:10.1111/j.1530-0277.2008.00807.x.

42. Guaiana G, Barbui C, Cipriani A. Hydroxyzine for generalised anxiety disorder. Cochrane Database Syst Rev. 2010;CD006815. doi: 10.1002/14651858.CD006815.pub2

43. Nirmalani-Gandhy A, Sanchez D, Catalano G. Terazosin for the treatment of trauma-related nightmares: a report of 4 cases. Clin Neuropharmacol. 2015;38:109-11. doi:10.1097/WNF. 0000000000000077.

44. Belendiuk KA, Babson KA, Vandrey R, Bonn-Miller MO. Cannabis species and cannabinoid concentration preference among sleep-disturbed medicinal cannabis users. Addict Behav. 2015;50: 178-81. doi:10.1016/j.addbeh.2015.06.032.
45. Garcia AN, Salloum IM. Polysomnographic sleep disturbances in nicotine, caffeine, alcohol, cocaine, opioid, and cannabis use: a focused review. Am J Addict. 2015;24:590-8. doi:10.1111/ajad. 12291.

46. Conroy DA, Arnedt JT. Sleep and substance use disorders: an update. Curr Psychiatry Rep. 2014;16:487. doi:10.1007/s11920-0140487-3.

47. Gates PJ, Albertella L, Copeland J. The effects of cannabinoid administration on sleep: a systematic review of human studies. Sleep Med Rev. 2014;18:477-87. doi:10.1016/j.smrv.2014.02.005.

48. Bonn-Miller MO, Babson KA, Vandrey R. Using cannabis to help you sleep: heightened frequency of medical cannabis use among those with PTSD. Drug Alcohol Depend. 2014;136:162-5. doi:10. 1016/j.drugalcdep.2013.12.008.

49. Babson KA, Boden MT, Bonn-Miller MO. The impact of perceived sleep quality and sleep efficiency/duration on cannabis use during a self-guided quit attempt. Addict Behav. 2013;38:2707-13. doi:10. 1016/j.addbeh.2013.06.012.

50. Babson KA, Boden MT, Harris AH, Stickle TR, Bonn-Miller MO. Poor sleep quality as a risk factor for lapse following a cannabis quit attempt. J Subst Abuse Treat. 2013;44:438-43. doi:10.1016/j.jsat. 2012.08.224.

51. Passie T, Emrich HM, Karst M, Brandt SD, Halpern JH. Mitigation of post-traumatic stress symptoms by Cannabis resin: a review of the clinical and neurobiological evidence. Drug Test Anal. 2012;4: 649-59. doi:10.1002/dta.1377.

52. Fraser GA. The use of a synthetic cannabinoid in the management of treatment-resistant nightmares in posttraumatic stress disorder (PTSD). CNS Neurosci Ther. 2009;15:84-8. doi:10.1111/j.17555949.2008.00071.x

53. Cameron C, Watson D, Robinson J. Use of a synthetic cannabinoid in a correctional population for posttraumatic stress disorder-related insomnia and nightmares, chronic pain, harm reduction, and other indications: a retrospective evaluation. J Clin Psychopharmacol. 2014;34:559-64. doi:10.1097/JCP.0000000000000180.

54. Jetly R, Heber A, Fraser G, Boisvert D. The efficacy of nabilone, a synthetic cannabinoid, in the treatment of PTSD-associated nightmares: a preliminary randomized, double-blind, placebo-controlled cross-over design study. Psychoneuroendocrinology. 2015;51:5858. doi:10.1016/j.psyneuen.2014.11.002. 